\title{
Abdominal obesity and cardiovascular disease
}

\begin{abstract}
There is no doubt that obesity has become a major disease in modern times and it is definitely associated with cancer, neurodegeneration and heart disease. Scientific studies have resulted in a growing consensus on the way abdominal obesity is associated with inflammation and cardiometabolic risk. Although the gender is a substantial factor of having abdominal fat, there are other protective factors including healthy eating and physical activity. Several techniques are used to assess obesity and their utilization depends on their feasibility and economic cost. This research is designed to address the important relationship between abdominal obesity and the risk of developing cardiovascular disease.
\end{abstract}

Keywords: abdominal obesity, metabolic syndrome, cardiovascular disease, body shape, inflammation, insulin resistance
Volume 3 Issue 2 - 2015

\author{
Rayan Saleh \\ Department of Food and Nutritional Sciences, University of \\ Reading, UK
}

Correspondence: Rayan Saleh, Registered Dietitian, Department of Food and Nutritional sciences, University of Reading, White knights, Reading, RG6 6AH, Berkshire, UK, Email rayanesaleh@gmail.com

Received: August 19,20I5 | Published:September 15, 2015
Abbreviations: WHO, world health organization; T2D, type 2 diabetes; BMI, body mass index; WC, waist circumference; WHR, waist to hip ratio; BIA, bio impedance analysis; DXA, dual energy x-ray absorptiometry; CT, computed tomography; MRI, magnetic resonance imaging; HDL, high density lipoprotein; LDL, low density lipoprotein; VLDL, very low density lipoprotein; SB, systolic blood pressure; DB, diastolic blood pressure; TG, triglyceride; FSA, food standard agency; VAT, visceral adipose tissue; CVD, cardiovascular disease; TOFI, thin on the outside fat on the inside; TNF- $\alpha$, tumor necrosis factor-alpha; IL-6, interleukin-6

\section{Introduction}

Obesity defined by a body mass index (BMI) of $30 \mathrm{~kg} / \mathrm{m}^{2}$ or higher, is an epidemic disease often correlated with positive energy balance due to social, economic, lifestyle and non modifiable factors. ${ }^{1}$ Globally, the prevalence of obesity has doubled between 1980 and 2008 and according to the World Health Organization (WHO), the United States of America has the highest proportion of obese and overweight people, while south Asia has the lowest percentage. ${ }^{2}$ Scientific studies have shown that Cardiovascular disease (CVD) has multiple risk factors, including unhealthy diet, physical inactivity, abdominal obesity, metabolic syndrome, type 2 diabetes (T2D), high levels of LDL (Low density lipoprotein) cholesterol and non modifiable factors such as age and genetics. ${ }^{3,4}$

Western diet based on meat, dairy and processed food high in fat, is positively associated with weight and CVD. A simple reduction of $5-10 \%$ of body weight has been demonstrated to improve metabolic profile and cardiovascular health. ${ }^{5}$ The FSA (food standard agency) in UK aims to decrease the intake of saturated fat from 13.3 to $11 \%$ and Trans fat to $2 \%$ of total fat intake. In fact, Trans fat (vegetable oils hydrogenated) put patients more at risk of developing CVD, by increasing the level of LDL (bad cholesterol) and reducing the level of HDL (good cholesterol) in blood. ${ }^{6}$ In addition to the important role of healthy diet on maintaining energy balance, physical activity can be the second major factor for managing obesity and CVD, not only by reducing visceral fat, improving metabolism activity and increasing HDL levels, but also by stopping the accumulation of ectopic fat.

There are several methods to assess obesity including anthropometric measurements (BMI, waist circumference WC, waist to hip ratio WHR), bioelectrical impedance analysis (BIA), Dual energy X-ray absorptiometry (DXA), Computed tomography (CT) and Magnetic Resonance Imaging (MRI). ${ }^{8}$ The gold standard method depends on the goal, the practicability and the economic cost. ${ }^{9}$ Simple non invasive anthropometric measurement such as BMI is shown to be associated with general obesity. ${ }^{10}$ Therefore, a better diagnosis of abdominal obesity for TOFI (thin on the outside fat on the inside) and Fat fit phenotypes consists of the combination of BMI with WC and WHR. TOFI are the people who have normal BMI $\left(<24.9 \mathrm{~kg} / \mathrm{m}^{2}\right)$ but high abdominal fat and thus more susceptible to T2D, while fat fit people are those with BMI $>30 \mathrm{~kg} / \mathrm{m}^{2}$ and are metabolically normal. ${ }^{11}$

\section{Metabolic syndrome and abdominal obesity}

A quarter of the world's adults have metabolic syndrome. There is evidence that people with metabolic syndrome have twice the risk of CVD and five times risk of T2D. ${ }^{12}$ Metabolic syndrome, syndrome $\mathrm{X}$ or insulin resistance syndrome is defined by the combination of 3 or more of those factors (Abdominal obesity, triglycerides TG $\geq 150 \mathrm{mg}$ / $\mathrm{dL}$, HDL (high density lipoprotein) cholesterol $<40 \mathrm{mg} / \mathrm{dL}$ in men or $<50 \mathrm{mg} / \mathrm{dL}$ in women, systolic blood pressure $(\mathrm{SB}) \geq 130 \mathrm{mmHg}$, diastolic blood pressure (DB) $\geq 85 \mathrm{mmHg}$, fasting plasma glucose $\geq 100 \mathrm{mg} / \mathrm{dL}$, glucose intolerance and insulin resistance. ${ }^{13}$

Abdominal obesity also known as central or visceral obesity is one of the essential characteristics of metabolic syndrome. There is a strong relationship between visceral fat (android obesity phenotype), insulin resistance and T2D. ${ }^{14}$ In addition, abdominal obesity is associated with CVD risk. People with high waist are at higher risk of CVD than those with smaller waist or those with different fat distribution around the hip and thighs rather than around the abdomen. ${ }^{15}$

Although women have more body fat percentage than men, they are at lower risk of cardiovascular disease. ${ }^{16}$ It is the distribution of fat which is related to heart health and not its weight. Women have a pear body shape characterized by Gynoid fat (fat located over the hips), or subcutaneous fat not shown to be related to heart disease. ${ }^{17}$ However, men have an apple body shape characterized by android visceral fat positively associated with CVD. ${ }^{18}$ For men, abdominal obesity is defined by a waist circumference (WC) $>102 \mathrm{~cm}$ (40 inches) and a wait to hip ratio (WHR) $>0.9$ and for women a WC $>88 \mathrm{~cm}$ ( 35 inches) and a WHR $>0.85$. For south Asian, WC should be higher than 
$80 \mathrm{~cm}$ ( 31.5 inches) in women and higher than $90 \mathrm{~cm}$ in men, to have abdominal obesity. ${ }^{19,20}$ Studies have shown that $1 \mathrm{~cm}$ increase in WC or 0.01 unit increases in WHR measurement were associated with $2 \%$ and $5 \%$ increase risk of cardiovascular disease respectively. ${ }^{20}$

Adipose tissue is believed to be an active endocrine proinflammatory organ (Figure 1). When adipose tissue reaches its maximum size, the excess of fatty acids coming from diet starts accumulating in ectopic sites (visceral adipose tissue, intrahepatic, intramuscular, renal sinus and pericardial tissue) leading to lipotoxicity. ${ }^{21}$ Adipose tissue produces healthy and unhealthy adipocytokines or adipokines. Although Adiponectin is believed to be a protective factor in regulating endothelial function, TNF alpha, Il6, resistin and visfatin are considered pro-inflammatory cytokines. ${ }^{22}$ Obese people show reduced plasma adiponectin levels and increased plasma levels of resistin, visfatin, TNF alpha and leptin. ${ }^{22}$ TNF alpha increases the risk of inflammation of oxidized complex macrophages-LDL, helping atherogenic plaque to form easily. ${ }^{23}$ Adipose tissue produces also non esterified fatty acids (FFA) in portal vein associated with the formation of VLDL (very low density lipoprotein) rich in TG and the formation of small dense atherogenic LDL which are susceptible to oxidation in the vascular wall. ${ }^{21}$ Although Leptin can have an important long term role in controlling appetite and regulating body weight, it is shown to be associated with inflammation, insulin resistance and cardiovascular disease risk. ${ }^{24-26}$

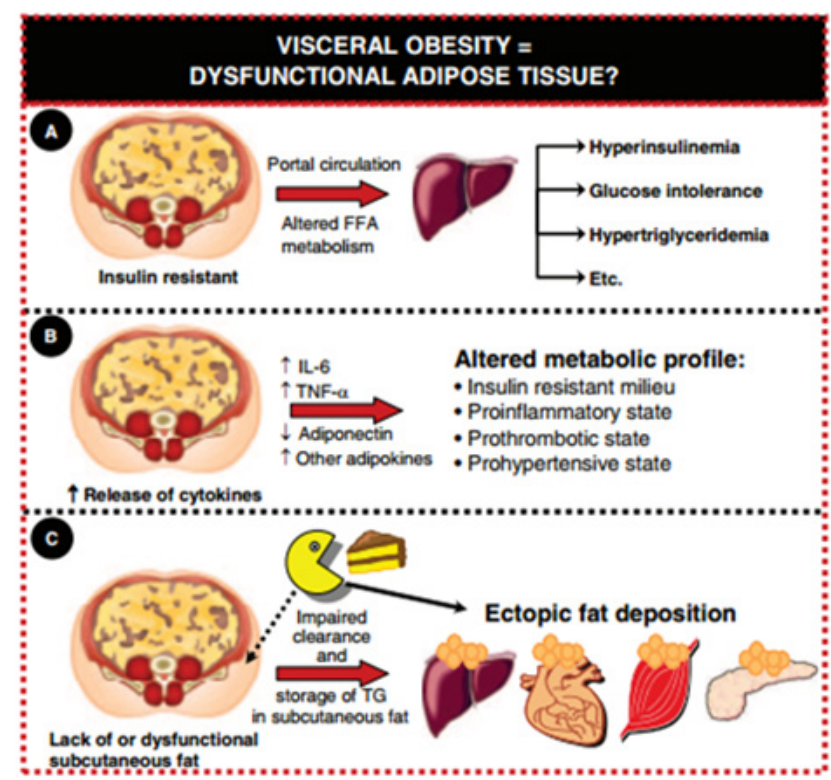

Figure I The mechanism viscceral obesity is associated with atherothrombotic-inflammatory abnormalities. ${ }^{14}$

${ }^{a}$ Visceral adipose tissue exposing the liver to FFA flux through portal vein, impairs glucose-insulin homeostasis leading to hyperinsulinemia and glucose intolerance.

${ }^{b}$ Adipocytokines and inflammation biomarkers secreted by visceral adipose tissu are responsible for metabolic abnormalities including insulin resistance, prothrombic and prohypertensive state.

'Excess fatty acids are stored as TG in ectopic sites (pericardial, intrahepatic tissue) rather than in adipose tissue.

\section{Conclusion}

In this short mini review, abdominal obesity is shown to be a major cause of death among CVD patients. The prevalence of obesity continues to increase, it is thus of great concern that more effort should be done in this field. Different methods are available to predict obesity risk at an early stage which would allow for intervention to reduce this epidemic disease. Although gender is a potential risk factor for abdominal obesity, genetic diversity of human population, diet and physical activity can also have a great impact. Considering all these factors in future studies can pose serious challenges for personalizing dietary advice as an efficient way to control obesity.

\section{Acknowledgements}

None.

\section{Conflict of interest}

The author declares no conflict of interest.

\section{References}

1. Balistreri CR, Caruso C, Candore G. The role of adipose tissue and adipokines in obesity related inflammatory diseases. Mediators Inflamm. 2010:2010:802078

2. Global Health Observatory (GHO): Obesity. World Health Organization; 2013.

3. Judith Mackay, George Mensah, Shanti Mendis, et al. The Atlas of Heart Disease and stroke. UK: Myriad Editions limited, World health organization in collaboration with the centers for Disease Control and Prevention; 2004. p. 1-116.

4. Salvatore M, Kristian BF, Jacques G, et al. The Metabolic Syndrome and Cardiovascular Risk a systematic review and meta-analysis. J Am Coll Cardiol. 2010;56(14):1113-1132.

5. Finelli C, Sommella L, Gioia S, et al. Should visceral fat be reduced to increase longevity? Ageing Res Rev. 2013;12(4):996-1004.

6. O Flaherty M, Flores-Mateo G, Nnoaham K, et al. Potential cardiovascular mortality reductions with stricter food policies in the United Kingdom of Great Britain and Northern Ireland. Bull World Health Organ. 2012;90(7):522-531.

7. Celis-Morales CA, Perez-Bravo F, Ibanez L, et al. Objective vs. Self-Reported Physical Activity and Sedentary Time: effects of Measurement Method on Relationships with Risk Biomarkers. PLoS One. 2012;7(5):e36345.

8. Rubenstein AH. Obesity: a modern epidemic. Trans Am Clin Climatol Assoc. 2005;116:103-111.

9. Gradmark AM, Rydh A, Renström F, et al. Computed tomography-based validation of abdominal adiposity measurements from ultrasonography, dual-energy X-ray absorptiometry and anthropometry. $B r J$ Nutr. 2010;104(4):582-588.

10. Vazquez G, Duval S, Jacobs DR, et al. Comparison of Body Mass Index, Waist Circumference, and Waist/Hip Ratio in Predicting Incident Diabetes: A Meta-Analysis. Epidemiol Rev. 2007;29:115-128.

11. Thomas EL, Fitzpatrick JA, Malik SJ, et al. Whole body fat: Content and distribution. Prog Nucl Magn Reson Spectrosc. 2013;73:56-80.

12. George Alberti, Paul Zimmet, Francine Kaufman, et al. The IDF Consensus definition of the Metabolic Syndrome in Children and Adolescents. ISBN 2-930229-49-7; 2007.

13. Grundy SM, Brewer HB, Cleeman JI, et al. Definition of Metabolic Syndrome Report of the National Heart, Lung and Blood institute/ American Heart Association Conference on Scientific Issues Related to Definition. Circulation. 2004;109(3):433-438. 
14. Després JP, Lemieux I, Bergeron J, et al. Abdominal Obesity and the Metabolic Syndrome: Contribution to Global Cardiometabolic Risk. Arterioscler Thromb Vasc Biol. 2008;28(6):1039-1049.

15. Samuel K. The case of visceral fat: argument for the defense. J Clin Invest. 2004;113(11):1530-1532.

16. Samsell L, Regier M, Walton C, et al. Importance of Android/Gynoid fat ratio in predicting Metabolic and Cardiovascular Disease risk in Normal weight as well as Overweight and Obese children. J Obes. 2014;2014:846578.

17. Després JP. Body Fat Distribution and Risk of Cardiovascular Disease: An Update. Circulation. 2012;126(10):1301-1313.

18. Aucouturier J, Meyer M, Thivel D, et al. Effect of Android to Gynoid Fat Ratio on Insulin Resistance in Obese Youth. Arch Pediatr Adolesc Med. 2009;163(9):826-831.

19. David York, Claude Lenfant. The Practical Guide Identification, Evaluation and treatment of overweight and obesity in adults. NHLBI Obesity Education Initiative, 2000. p. 1-80.

20. Lu Q, Ma CM, Wang R, et al. Value of dual-energy X-ray absorptiometry derived parameters vs anthropometric obesity indices in the assessment of early atherosclerosis in abdominally obese men. Obes Res Clin Pract. 2012;6(4):e263-e346.
21. Bastien M, Poirier P, Lemieux I, et al. Overview of Epidemiology and Contribution of Obesity to Cardiovascular Disease. Prog Cardiovasc Dis. 2014;56(4):369-381.

22. Uslu S, Kebapçi N, Kara M, et al. Relationship between adipocytokines and cardiovascular risk factors in patients with type 2 diabetes mellitus. Exp Ther Med. 2012;4(1):113-120.

23. William PC, Jaswinder KS. TNF- $\alpha$ and adipocytes biology. FEBS Letters. 2008;582(1):117-131.

24. Havel PJ. Role of Adipose Tissue in body-weight regulation: mechanisms regulating leptin production and energy balance. Proc Nutr Soc. 2000;59(3):359-371.

25. Hukshorn CJ, Lindeman JH, Toet $\mathrm{KH}$, et al. Leptin and the Proinflammatory State Associated with Human Obesity. J Clin Endocrinol Metab. 2004;89(4):1773-1778.

26. Sada Y, Katabami T, Asai S, et al. Intrahepatic lipid content is linked to insulin resistance in obese subjects. Obes Res Clin Pract. 2011;5(2): 79 e156. 